\title{
A Supporting Enterprise Infrastructure Using STEP Technologies
}

\author{
S.P.Reid \& Dr.S.K.Banerjee \\ University of Strathclyde \\ DMEM, James Weir Building, 75 Montrose Street, Glasgow, G1 $1 X J$ \\ Telephone: +44 (0) 1415482065
}

Fax: +44 (0)1415520557

E-Mail: s.k.banerjee@strath.ac.uk

\begin{abstract}
Many organisations have evolved into disparate functional units through the adoption of a decentralised control structure. Meaningful and effective exchanges of information between these units are required to support organisational integration. This paper proposes the use of STEP technology and software for implementing an integrated information system that can act as a supporting infrastructure to the organisation's functional units. The paper describes a methodology, using a case example, that can be used to develop such an infrastructure.
\end{abstract}

\section{Keywords}

Enterprise modelling, Information Infrastructure, STEP, Express.

\section{INTRODUCTION}

Manufacturing industries are experiencing a market place dominated by supply, where time based competition is now a reality, and 'time to market' is the challenge for manufacturing management. In order to deal with the ever increasing environmental choices and uncertainties related with manufacturing a more flexible, responsive and creative management is required. Today's competitive organisation is dynamic and constantly changing; unless this change is effectively managed organisations may experience difficulties and future survival. The two main aspects of change that have impacted the organisation are the rapid growth in the number 
of products and services, and the speed with which new products and services invade the marketplace. When customer demand patterns are considered in conjunction with this, the management of manufacturing becomes complex and requires a system that is flexible enough to respond quickly to changes and has the appropriate variability handling capability. This flexibility of a system can be provided in the design stage by incorporating the required level of flexibility of resources into the system, and by using rules, procedures and guidelines during the systems operation in order to minimise the effect of change during manufacture (Duimering et al 1992). This implies that to manage today's manufacturing system a more dynamic approach to the design and operation of an organisation is required.

In attempting to become more dynamic in their operation and while trying to improve their overall approach to efficiency, many organisations have adopted decentralised structures and evolved into disparate and autonomous departments or units. However, in trying to optimise the functional efficiency of a unit, one may lose sight of the strategies and goals of the organisation as a whole. Co-ordination of these units therefore is a primary concern in this respect.

Enterprise Integration is a relatively new concept that is trying to address the problems of modern enterprises by integrating different and disparate organisational functions. Enterprise Integration can be defined as the enhancement of the co-ordination between organisations, systems and individuals, that in doing so improve the performance of some of the participating parts, whilst not decreasing that of any. The main goal of enterprise integration is to promote the success of the business itself; its effectiveness being judged by how well the separate enterprise components co-operate with each other to achieve the stated goals of the organisation as a whole. From this point of view it can be seen that there is a need for effective communication through an integrating infrastructure that would link the disparate departments.

It is possible to develop an integrating infrastructure in a particular area of interest to aid systems managers by using what is available or known at present. Such an infrastructure could be created using a neutral data format so that it will enhance the process of information management and transfer between the participating units of a system. Having such an infrastructure would increase the availability and ease of flow of appropriate information between the departments even with different computer applications running on differing hardware platforms.

\section{2 \\ MODELLING THE ENTERPRISE}

An essential part of the integration process is the creation of a valid enterprise model. A comprehensive review of issues regarding enterprise modelling can be found in Patankar and Adiga (1995). An enterprise model will represent the components of an organisation, their function, their interactions and the information flow between them. This enterprise model can then be used as the basis for the creation of the integrating infrastructure. There have been three methods suggested for creating enterprise models and these are (Petrie 1992);

- The Master Model approach,

- Unification, and

- The Federated approach. 


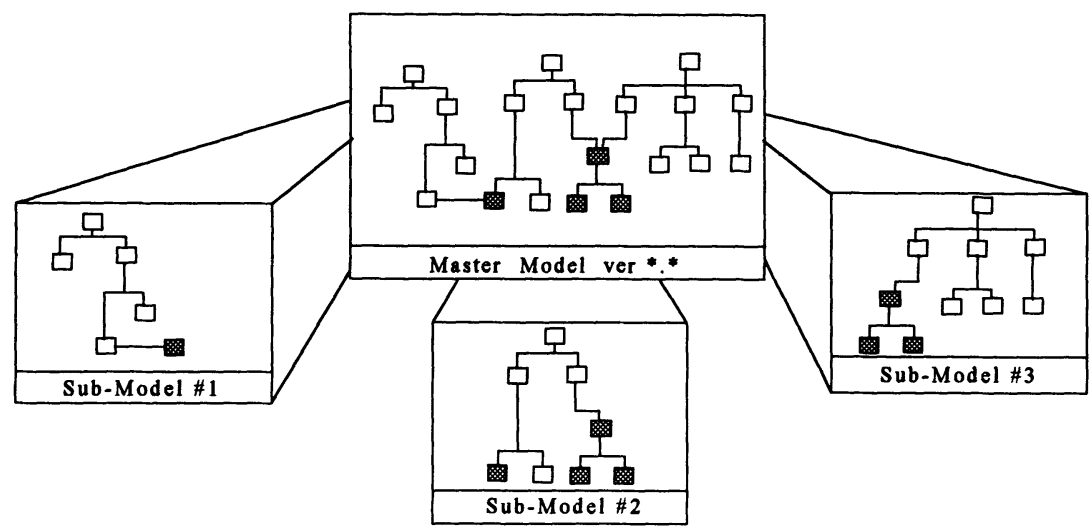

Figure 1 Sub-models can be derived / extracted from the Master model.

The authors have adopted the master model approach; this approach rests on creating a single reference model from which it is possible to derive and extract sub-models that represent the required views of the organisation from different perspectives. This principle can be seen in Figure 1. The IEM concept (Mertins et al 1992) also follows this approach.

In order to create an enterprise infrastructure that will bring together disparate organisational functions it is necessary to create accurate models that can be used to represent the different functional areas of the organisation. Such functional models can be created using a neutral information modelling language, such as Express and then can be linked, through their commonalities, to form a larger 'Master Model'; a methodology also advocated by Hars and Scheer (1992). It can then be used to create valid instances of the companies' requirements.

The developed master model may be used as the basis for defining the structure of an object oriented database implementation that will hold the organisations data in a convenient form and location, and from which users can access the information they require.

However, before this can be done the important functional aspects and entities within the organisation have to be identified so that nothing of importance is left out of the models. The process of identifying these important organisation qualities is covered during the requirement's analysis phase. 'The determination of the requirements of the organisation is an integral part of the modelling process as it involves creating a set of requirements that fully represent the company's needs and each unit's contribution to them without overlooking any particular aspect of the company. It is therefore imperative that there be a high level of communication between developers and management during the requirement's analysis phase.

The requirements, and hence the models, are developed through discussing the problem domain with the people who are most familiar with it, e.g. those responsible for activities and information, as well as the users of the information (van Griethuysen 1992). Methodologies such as Quality Function Deployment (QFD) could be used to create such a set of requirements. 


\section{3}

\section{ENTERPRISE MODELLING USING EXPRESS}

Having completed the requirements phase the Express information modelling language (Schenck and Wilson 1994) can be used for creating models of an organisation's information system. This modelling language is now an international standard that has been developed as part of the STEP ${ }^{1}$ standard and its formal structure is defined in (ISO 1991). Express was originally designed for the purpose of creating models that could be used to facilitate the exchange of data concerning mechanical products. The scope of its use has broadened considerably since.

The Product Data Exchange Specification (PDES) provides a way of representing product data in a neutral format throughout the products life cycle. PDES has three important components (Qiao et al 1993):

\section{- Reference Models, \\ - The Express modelling language, \\ - The STEP file structure.}

The development work within the STEP domain has been conducted within a variety of areas. These development areas are commonly referred to as 'Parts' within STEP, the number of which is continually growing. Each of these parts contains information relating to a particular aspect of the standards for product representation and description and usually incorporate reference models that have been developed using Express.

The whole basis of STEP is, as its name states, to define the standard for the exchange of product model data, and it is with this in mind that most of the available STEP related tools have been developed. The authors believe, however, that it is reasonable to use these technologies for the purpose of creating an enterprise infrastructure that will encompass and support the needs of the whole organisation.

The Express information modelling language was chosen for use in the model development phase for the following reasons:

- It is now an international standard information modelling language, defined in ISO 10303 , and has a large and growing user base.

- It is computer interpretable, and tools are available that can be used to validate and manipulate Express models. There are tools available, called Express Parser's, which can be used to check an Express models' semantic and syntactic correctness. Examples of these include Fedex and ICE methods, developed by NIST $^{2}$ and The CadLab at The University of Paderborn

- There is a wide and increasing variety of tools available in the market place as well as in the public domain that can be used for the development of Express information models and the data instances for them. A comprehensive list of Express tools and services can be found in (Wilson 1995).

It uses a neutral data storage format, i.e, the STEP file format, which greatly enhances the ability to exchange data between applications readily. The ISO 10303-21 (ISOb 1991) constrains the number of instances within a single STEP file to $10^{9}-1$ instances (Lehrenfeld et al 1994).

- It has a graphical subsection in its definition that allows for models to be developed using graphical tools as opposed to just text editors. This greatly increases the ability of

1 STEP is the Standard for the Exchange of Product model data.

2 NIST is The National Institute for Standards and Technology. 
developers to visualise a model and its inter-relations as well as to edit and update Express models.

Following the Master model approach, the use of Express brings some distinct advantages to the modelling process. It is possible for the master model to evolve over time so as to encompass the whole of the organisation. This makes it possible for the model development process to be conducted in an incremental manner, if required, where organisational units or departments are modelled and then incorporated into the Master model.

The use of a single model format facilitates the integration process as new models can make use of the developed model and can therefore incorporate any commonalities that already exist in the model. This has the effect of reducing the problem associated with semantic integration as there will only be one representation of each requirement within the model. The viewpoint for a particular department may require information concerning an entity that is not required by any other departments. This information can be masked from other viewpoints by not including the attributes in the extracted model. This does not mean that the information will not be there; it will just not be visible to those who do not require that particular information.

In order to overcome consistency problems between model formats, Felser and Mueller (1994) propose an extension to the Express modelling language that allows the modelling of processes. The proposed extensions include;

- interface declarations,

- local functions and procedures,

- $\quad$ system and process declarations, which are all placed within the existing entity declaration. While this extension will not conflict with the current Express specification (ISOa 1991), there will be problems when trying to validate these models with existing STEP tools.

\section{DEVELOPING THE INFRASTRUCTURE}

The process of creating a supporting infrastructure, as shown in Figure 2, starts with the organisation conducting a requirement's definition exercise that will yield the data and information flows that are critical to the organisations functioning. This will also involve identifying information that is needed by specific users and departments within the system (functional units). The requirements generated can then be used to create an Express model of the organisations functional requirements. This can be done by making use of an Express-G graphics package. This will allow the user to create and link entities, which will represent the real world objects that are of interest, to form an enterprise model. By using the Express-G package the model developers will be able to edit with ease and update the enterprise model to incorporate all of the required information. The Express- $G$ package generates the Express code corresponding to the developed enterprise model. This can then be validated by an Express Parser that will check the model against the definitions and constraints defined within the Express modelling language (ISO 10303-11). If there are any errors in the model they will be identified and the model can be modified to erase them.

When the developers are happy that they have a model that accurately represents their system and includes all the information that may be required by the system users, the enterprise model can become the master model. Preliminary work has been conducted into creating a supporting infrastructure that utilises the NIST Data Probe software for creating and editing instances of the manufacturing system (Reid and Banerjee 1995). This system use importing 


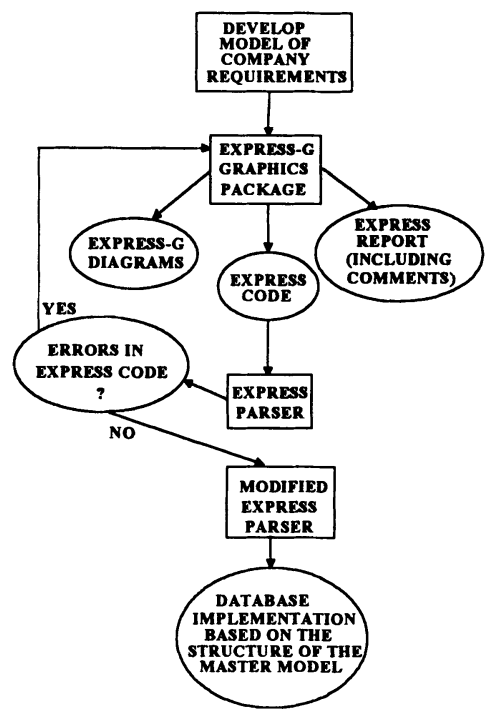

Figure 2 Outline of the process of creating a. supporting infrastructure.

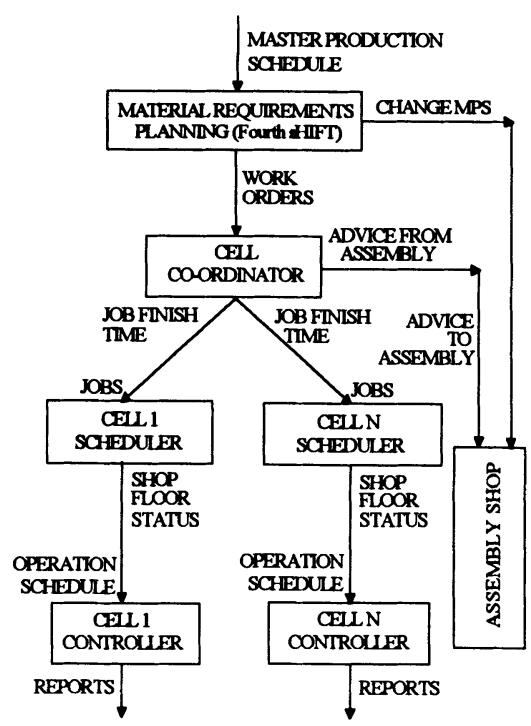

Figure 3 Structural overview of the production planning and control system.

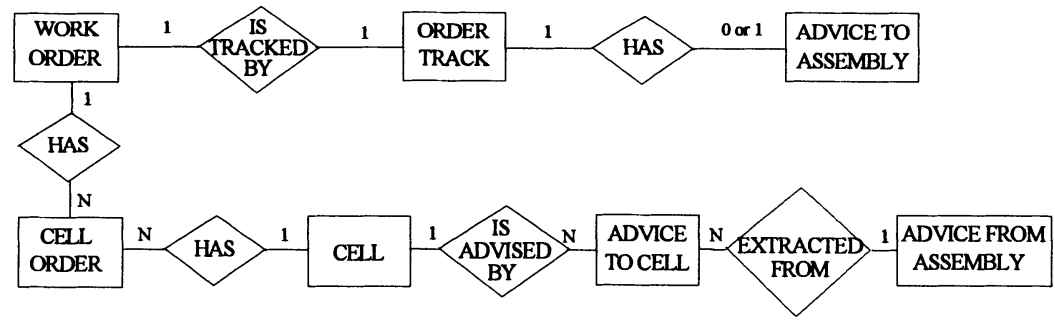

\begin{tabular}{|c|c|c|c|c|}
\hline WORK ORDER & ORDER TRACK & CELLORDER & ADVICE TO ASSEMBLY & ADVICE TOCEL \\
\hline WORK ORDER NUMBER & WORK ORDER NUMBER & WORK ORDER NUMBER & WORK ORDER NUMBER & CEU LNUMBER \\
\hline PART NUMBER & PART NUMBER & PART NUMBER & DATE & DATE \\
\hline ORDER QUANTITY & CEL L NAME & CEL NUMBER & ADVICE & ADVICE \\
\hline START DATE & FINISH DATE/TIME & & & \\
\hline DUE DATE & AUOCATEDFLAG & CDLL & \multirow{2}{*}{\multicolumn{2}{|c|}{$\begin{array}{l}\text { ADVICE FROM ASSEMBLY } \\
\text { SEQUENCE NUMBER OF ADVICE } \\
\text { DATE } \\
\text { ADVICE }\end{array}$}} \\
\hline ORDER STATUS & $\begin{array}{l}\text { START DATE } \\
\text { DUE DATE } \\
\text { ORDER STATUS } \\
\text { LATE FLAG }\end{array}$ & $\begin{array}{l}\text { CEU NUMBER } \\
\text { CEIL NAME } \\
\text { CEL D DESCRIPTION } \\
\text { CEU L MANAGER }\end{array}$ & & \\
\hline
\end{tabular}

Figure 4 Entity relationship diagram with attributes for Cell Co-ordinator data model. 


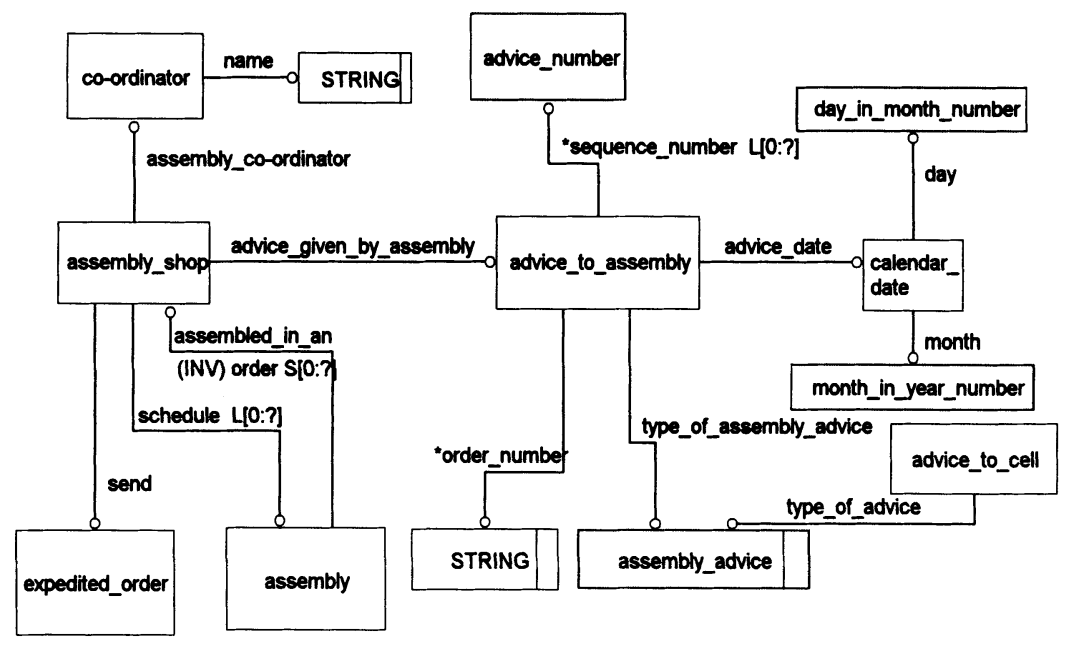

Figure 5 Entity level diagram in Express-G, of the Assembly schema, for use with the Data Probe software.

and exporting STEP files as its means for updating the information in different viewpoints of the manufacturing system.

A model of a manufacturing planning and control system was developed for a manufacturing company (Zhou 1993), the structure of which is shown in Figure 3. This system was originally defined using entity relationship diagrams, as in Figure 4 and data flow diagrams, which were subsequently translated into Express; an example is shown in Figure 5.

Although the arrangement developed through the preliminary work for editing and updating the manufacturing system information may be adequate for some implementations, an implementation that made use of the STEP Standard Data Access Interface (SDAI ${ }^{3}$ ) and a database system would increase the usefulness and applicability of the infrastructure. To this end the master model can be used to create an implementation of an object oriented database. This is done by modifying an express parser so that it accepts an express model, in this case the master model, and outputs the implemented database structure which is a mapping from the master model into the database system (Luhrsen and Krebs 1995). The modifications required to the express parser depend upon the particular database system being used. This is commonly known as creating a 'back-end' to the parser. The structure of the Express modelling language lends itself well to being translated into databases that are $\mathrm{C}++$ based as they have similar data structures.

The next stage in the development process is to create an implementation of the STEP SDAI. The SDAI is used to control the access to information relating to specified express models. Implementations of the SDAI may involve the manipulation of STEP files and their data or the manipulation of a database and its information. In the case described the structure of the database is defined by the structure of the master model. It can be seen in Figure 5 that if

3 The SDAI is defined in STEP Part 22, and a $\mathrm{C}++$ binding to the SDAI is defined in Part 23. 
a user's information requirements are defined by a sub-model of the master model, then the information held within the database relevant to the end-user will correspond to the same sub-model. Therefore the SDAI will provide the end-user access to the information based on their specific sub-model. The actual data that the end-user will be able to edit and update will be defined during the database user set-up procedures. The ownership and access of data are therefore predefined.

In order to be able to provide the required information to the end-users, when they need it, the SDAI must have some means of communicating with the database. The SDAI does this by having function calls to the database systems manager (DBMS) incorporated into its definition (Goh et al 1994, Rando and McCabe 1994). Therefore, if the end-user wants to query the database they initiate the query at the SDAI interface, which then calls the database management system. The database management system interrogates the database and returns the required information to the user. This process can be seen in Figure 6 . Because the implementation details of the SDAI have not been defined it is possible for all SDAI implementations to have the same definition and user interfaces, no matter what database

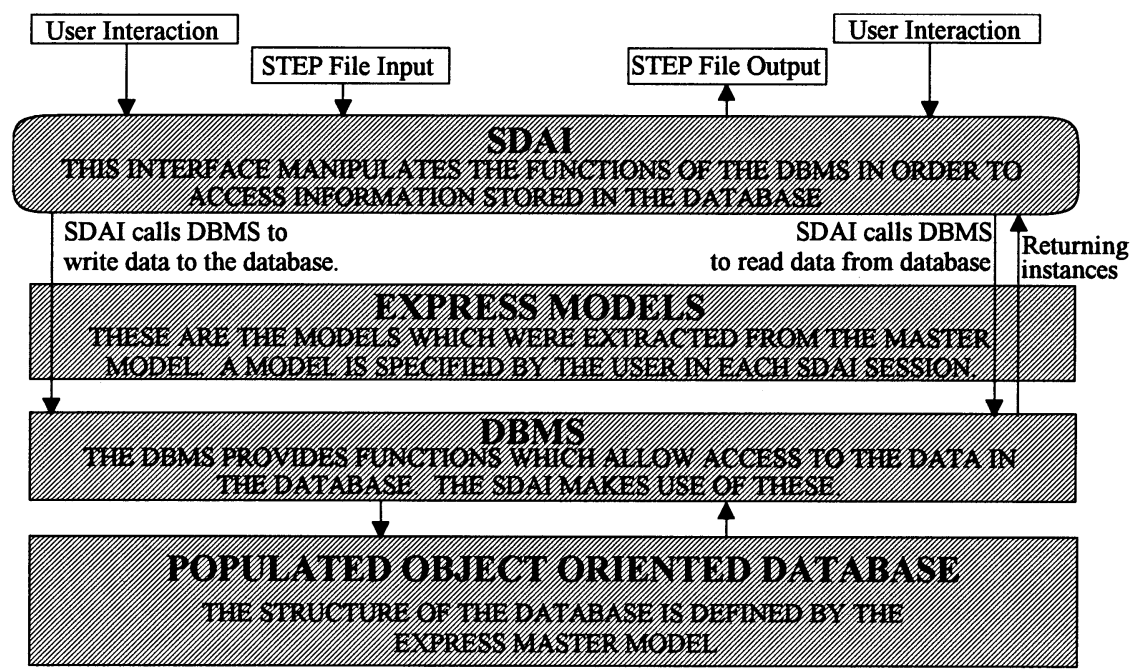

Figure 6 Query initiation and response process using an SDAI implementation together with an object-oriented database and management system.

system they are implemented to.

With the supporting infrastructure developed and the SDAI implemented it is possible for company specific programs to be written that will provide the end-user with the information they require when they need it. These programs will be extensions to the SDAI user interface, in that they will be connected to the SDAI, but the end-users' display will show the information that is needed in the required format, accessed via the SDAI. In this fashion the information obtained by the SDAI is manipulated into a form that is most suitable for the specific end-user. 


\section{CONCLUSIONS}

In today's dynamic working environment it is necessary for organisations to have a supporting information infrastructure that will reduce information duplication and redundancy, while providing the end-user the information they require. A reconfigureable infrastructure like this can be created using current STEP technologies. The research undertaken by the authors has shown that it is possible to use these current tools for the creation of a supporting information infrastructure that allows updating of information from different enterprise viewpoints. It can also be noted that the introductory period for gaining knowledge on the use of these tools is very short. The authors are currently investigating the use of a SDAI implementation with an object-oriented database as a natural progression in this area of research.

While there is no scope for modelling processes within the current ISO definition of Express we believe that the modelling of processes and change should be tackled in another manner. While the information within a system may change its state there is no mechanism within Express to conduct this change automatically. Therefore, to be able to create a supporting infrastructure that can adapt to these changes it is necessary to develop procedures that can be used to change the state of instances within a database. The authors are investigating the use of object-oriented methods, such as Booch (1994), for providing the facilities for the definition of programs which will then be able to manipulate the stored data. These programs will be developed from the information requirements of the end-users and will allow the information to be presented in a usable form when the users require it.

Ellis et al (1994) proposes a similar approach for describing a reference model for open distributed processing (RM-ODP), where the information view is described using IDEF0 and Express, and a computation view is described using Booch diagrams.

Using the methodology outlined in this paper it is possible to create a supporting infrastructure for an organisation that can be extended to include more aspects of the organisation as and when they are modelled. The use of STEP tools allows for the rapid reconfiguration of viewpoints, or sub-models, of the Master model of the organisation.

\section{REFERENCES}

Duimering, R. P., Safayeni, F., Purdy, L., 1992, Future factories and today's organisation. 8th International Conference, CARS \& FOF, Metz France, August 17-19

Ellis., T.I.A., et al, 1994, The development of an information sharing platform for concurrent engineering. Proceedings of the European Workshop on Integrated Manufacturing Systems Engineering (IMSE '94). Grenoble, France, December 12-14.

Felser, W., Mueller, W., 1994, Express-P - Extending Express for process modeling and monitoring. ASME Computers in Engineering Conference, Minneapolis, MI. September 11-14.

Goh, A., Hui, S. C., Song, B., Wang, F. Y., 1994, A study of SDAI implementation on object-oriented databases. Computer Standards \& Interfaces, 16(33-43).

Hars, A., Scheer, A-W., 1992, Reference models for enterprise wide data engineering. Proceedings of the 1st International Conference on Enterprise Integration Modeling. (MIT Press). 
ISOa, 1991, ISO 10303-11 : Industrial Automation Systems And Integration - Product Data Representation And Exchange - Part 11 : Description Methods : The Express Language Reference Manual.

ISOb, 1991, ISO 10303-21 : Industrial Automation Systems And Integration - Product Data Representation And Exchange - Part 21 : Implementation Methods : Clear text encoding of the exchange structure.

Krebs, T., Luhrsen, H., 1995, STEP databases as integration platform for concurrent engineering. Proceedings of the 2nd International Conference on Concurrent Engineering. McClean, Virginia, August 23-25, pp.131-142

Lehrenfeld, G., Mueller, W., Wiechers, N., 1994, Parallel validation of STEP files. Proceedings of the 2nd International Conference on Concurrent Engineering \& Electronic Design Automation (CEEDA '94). Poole, UK, April 7-8.

Mertins, K., Sussenguth, W., Jochem, R., 1992, An object oriented method for integrated enterprise modelling as a basis for enterprise co-ordination. Proceedings of the lst International Conference on Enterprise Integration Modeling. (MIT Press).

Patankar, A. K., Adiga, S., 1995, Enterprise integration modelling: a review of theory and practice. Computer Integrated Manufacturing Systems. Vol.8, No.1, pp21-34

Petrie, C. J., 1992, Introduction. Proceedings of the 1st International Conference on Enterprise Integration Modeling. (MIT Press).

Qiao, L-H., Zhang, C., Liu, T-H.,, Wang, B., Fischer, G. W., 1993, A PDES/STEP based product data preparation procedure for computer-aided process planning. Computers in Industry. Vol.21, Part 1, pp.11-22.

Rando, T., Mccabe, L., 1994, Issues in implementing the $C++$ binding to the SDAI. Computer Standards \& Interfaces, 16(331-340).

Reid, S. P., Banerjee, S. K., 1995, Information system for manufacturing. Proceedings of the European WorkshopDynamic Control and Management Systems in Manufacturing Processes, October,Prague, ppl4.

Schenck, D. A., Wilson, P. R., Information Modeling : The Express way. (Oxford University Press Inc, New York).

Van Griethuysen., J. J., 1992, Enterprise modelling, a necessary basis for modern information systems. Open Distributed Processing. IFIP Elsevier Science Publishers B.V., J. de. Meer, V. Heymer \& R. Roth (Eds).

Wilson, P. R., 1995, Express Tools and Services. NIST, Building 220, Room A127, Gaithersburg, MD 20899.

Zhou, M., 1993, Design and development of PPC systems for AMS using structured methodologies and CASE tools. Unpublished $\mathrm{PhD}$ thesis, University of Strathclyde, UK.

\section{BIOGRAPHY}

S.P.Reid is a $\mathrm{PhD}$ research student at the University of Strathclyde whose background is in Mechanical Engineering, and his current research work is in STEP technologies for manufacturing support system development.

Dr.S.K.Banerjee is a senior lecturer at the University of Strathclyde and is a member of the task force on Enterprise Integration, IFAC/IFIP WG 5.12. 